\title{
THE EFFECTS OF GENTAMICIN-IMPREGNATED COLLAGEN SPONGE VERSUS GENTAMICIN-IMPREGNATED POLYMETHYLMETHACRYLATE BEADS IN PATIENTS WITH OSTEOMYELITIS
}

\author{
AHMAD ARIEF ATAN, MOHD YAZID BAJURI*, ABDUL MUHAIMIN ALI, RASYIDAH REHIR, \\ AHMAD FAZLY ABD RASHID
}

Department of Orthopaedics and Traumatology, Universiti Kebangsaan Malaysia Medical Centre, Kuala Lumpur, Malaysia. Email: ezeds007@yahoo.com.my

Received: 03 May 2018, Revised and Accepted: 30 July 2018

\section{ABSTRACT}

Objective: The usage of antibiotic-impregnated polymethylmethacrylate (PMMA) beads is regarded as the gold standard for local antibiotic delivery. However, the relatively new antibiotic-impregnated collagen sponge has multiple advantages over it. The objective of this study is to compare the measurable effects between gentamicin-impregnated collagen sponge and gentamicin-impregnated (PMMA) beads in patients with osteomyelitis.

Methods: This is a case-control study which involved 60 patients who were diagnosed with osteomyelitis between January 2014 and June 2015 , and underwent first surgical debridement with application of either gentamicin-impregnated collagen sponge (n=28) or gentamicin-impregnated PMMA beads $(n=32)$. The numbers of debridement, trend of blood parameters, duration of hospitalization, and total duration of systemic antibiotic therapy needed to be completed were reviewed from the patients' file.

Results: A total of 53 patients of 60 were diagnosed with osteomyelitis of the lower limbs, while the remaining seven were involving the upper limbs. The gentamicin-impregnated collagen sponge group has significant lower reoperative rate $(\mathrm{p}<0.05)$ and also significant reduction of total white cell count in 6 weeks $(\mathrm{p}<0.05)$. The two groups showed no statistical difference in regard of duration of hospital admission, duration of systemic antibiotic therapy completed, and the reduction of C-reactive protein at 6 weeks post-debridement.

Conclusion: This study reiterates the efficacy of gentamicin-impregnated collagen sponge that results in lower reoperative rate as compared to conventional gentamicin-impregnated PMMA beads.

Keywords: Osteomyelitis, Local antibiotics, Gentamicin impregnated, Collagen sponge, Polymethylmethacrylate beads.

(C) 2018 The Authors. Published by Innovare Academic Sciences Pvt Ltd. This is an open access article under the CC BY license (http://creativecommons. org/licenses/by/4. 0/) DOI: http://dx.doi.org/10.22159/ajpcr.2018.v11i12.26910

\section{INTRODUCTION}

Osteomyelitis is still a socioeconomic burden due to multiple factors including the increasing numbers of road vehicle accidents, which leads to increase incidence of fractures and post-traumatic osteomyelitis; the high numbers of diabetic and obese patients which predispose to osteomyelitis and emergence of multiple drug-resistant organisms [1-3].

Osteomyelitis is loosely defined as infection of the bone and its marrow, notable with the progressive inflammatory destruction and apposition of new bone following it. It begins with the spread of the bacteria, either by hematogenous seeding, direct inoculation, airborne contamination, or contiguous focus route. The subsequent contact with bone facilitates cell-to-cell or cell-to-implant adhesion, and the organism becomes sessile and reduces metabolic rate. Biofilm produced by the organism further protects them from phagocytosis, opsonization, and antimicrobials [4]. Disruption of local blood supply ensues, with the sequelae of formation of sequestrum and involucrum. The pathogenesis above outlined various factors on why managing osteomyelitis is an uphill challenge. Despite adequate debridement and commencement of culture-sensitive antibiotics, multiple factors above provide barriers for success in managing osteomyelitis [5]. The failure systemic antibiotics to the affected sites with suboptimal debridement allow organisms to not only colonize and replicate but also to be dormant. Therefore, resolution of acute infection will usually precede the development of chronic osteomyelitis.

Hence, the management of osteomyelitis needs to be optimized in all possible angles including the usage of local antibiotics remains as one of the important measures of treatment of osteomyelitis. In this study, we are trying to explore a small part of the multiple facets of the local antibiotics delivery in patients with osteomyelitis. We are focusing on two types of local antibiotics - the antibiotic-impregnated collagen sponge, which is relatively new but with many advantages, and the antibiotic-impregnated polymethylmethacrylate (PMMA) beads, which has been accepted as the standard of local antibiotics delivery in orthopedic.

As PMMA beads exist in the form of chains with solid beads, there will be the need to remove it after its application. Because of this, we have hypothesized that the usage of antibiotics impregnated collagen sponge will render the patients to have lower reoperative rate, as the collagen sponge will dissolve over time. We have also hypothesized that due to this, the hospitalization stay will be shorter for them. This may provide a platform to strategy on the direction of resources in the management of osteomyelitis.

\section{METHODS}

This was a retrospective, case-control study involving 60 patients who were diagnosed with osteomyelitis between January 2014 and June 2015 in Malacca General Hospital, Melaka, Malaysia. All of these patients underwent their first surgical debridement after the diagnosis with the application of either gentamicin-impregnated collagen sponge or gentamicin-impregnated PMMA beads.

The patients were first screened based on the documentation in admission log in wards, surgery census in operation theater, and outpatient visit log in Orthopaedic Outpatient Clinic, Malacca General Hospital. Patients that 
were subjected to debridement with application of only one type each for gentamicin-impregnated collagen sponge and gentamicin-impregnated PMMA beads were selected. For the collagen sponge, the brand used was Collatamp with the size of $10 \mathrm{~cm}$ by $10 \mathrm{~cm}\left(1 \mathrm{~cm}^{2}\right.$ contains equivalent of $1.3 \mathrm{mg}$ gentamicin base); whereas for the PMMA beads, single chain of the Septopal of 30 beads per chain was used (each bead contains equivalent of $4.5 \mathrm{mg}$ gentamicin base). This is to ensure both have almost similar content of gentamicin to reduce the variable bias. Both were purchased by the Orthopaedic Department of Malacca General Hospital, and patients were not required to pay for any of them.

Exclusion criteria were patients who defaulted follow-up before the resolution of infection or non-compliant to oral antibiotics or blood taking. Other exclusion criteria include patients who sustained polytrauma or have significant comorbidity that may affect healing process. Severely immunocompromised patients were also not selected into the study.

Then, the patients' data were collected using their admission records from Unit Rekod Perubatan, Malacca General Hospital and their outpatient notes from Orthopaedic Outpatient, Clinic Malacca General Hospital. Apart from the demographic data, these patients' blood parameters were collected, especially the C-reactive protein (CRP) and total white cell count (TWC) levels which were recorded at the time of diagnosis and at least every 2 weeks afterward until the resolution of the infection. The length of hospital stay, the numbers of subsequent admission and debridement, and the total duration of antibiotics were also recorded.

\section{RESULTS}

From the 60 patients included in this study, 52 were male and 8 were female. Patients' age ranged between 17 and 70 years old with the average of 32.9 years old. All of these patients underwent follow-up in the Outpatient Orthopaedic Clinic, Malacca General Hospital, for the minimum of 1 year and 5 months. All patients were diagnosed with osteomyelitis, with 53 involving the osteomyelitis of lower limbs and seven patients with osteomyelitis of the upper limbs. For the lower limbs group, 20 patients had osteomyelitis of the femur, 22 patients had osteomyelitis of tibia or fibula or ankle, and one patient had osteomyelitis of the patella. For the upper limbs group, only one had osteomyelitis of humerus while the other six involved radius and/or ulna (Fig. 1).

Of the 60 patients, 32 had an implant at the site of osteomyelitis during the time of diagnosis, whereas 19 were on external fixator and the remaining nine have neither an implant nor external fixator. Of the 32 patients with implant, 27 had their implant removed during the first surgical debridement after the diagnosis of osteomyelitis. Further, analysis revealed that the reoperative rate is statistically higher in the patients who underwent surgical debridement with application of gentamicin-impregnated PMMA beads (Table 1).

In overall, for the collagen sponge group, the maximum number of debridement was 4 (mean 2.04), whereas for the PMMA beads group, it was 6 (mean 2.88). Using the Mann-Whitney U-test, the collagen sponge group has a statistically significant lower number of debridement than the PMMA beads group with $\mathrm{p}=0.005$. When comparing the duration of hospitalization for both groups, the collagen sponge group has shorter duration of hospital stay (mean 24 days) compared to the PMMA beads group (mean 31 days); however, this is not statistically significant ( $p>0.05$ ) (Table 2).

The two blood parameters analyzed in this study were the CRP and TWC count, as both are considered as reliable markers for inflammation and assessment of response to treatment. Both parameters trend were then compared based on the level taken at the time of diagnosis and level taken at 6 weeks after the debridement with application of the local antibiotics (collagen sponge or PMMA beads).

There is no significant difference between the two groups in terms of the reduction of CRP at the $6^{\text {th }}$ week after the debridement $(p>0.05)$ (Fig. 2 and Table 3).

However, there was difference between the two groups in terms of the trend of reduction of TWC. Patients who underwent debridement with application of gentamicin-impregnated collagen sponge were shown to have significant reduction of TWC when compared to the PMMA beads group. With correction of the violated assumption of sphericity done using GreenhouseGeisser correction, $\mathrm{p}$ value was $0.02(\mathrm{p}<0.05)$ (Fig. 3 and Table 4).

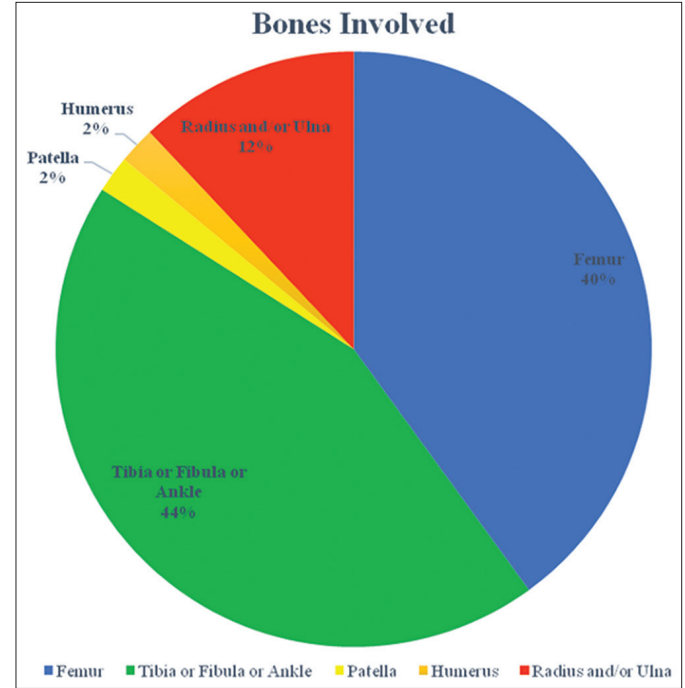

Fig. 1: Distribution of osteomyelitis according to the type of bones involved

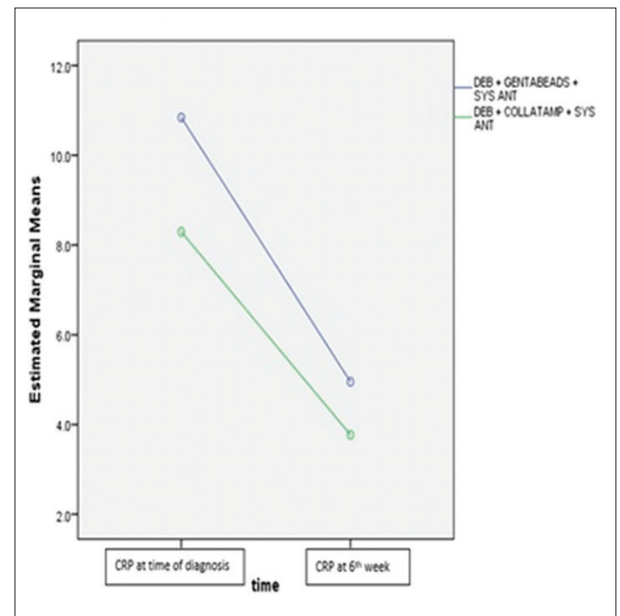

Fig. 2: C-reactive protein trend between the time of diagnosis and at 6 weeks after debridement

Table 1: Reoperative rate between patients who underwent debridement with gentamicin-impregnated collagen sponge and PMMA beads

\begin{tabular}{llll}
\hline Type of treatment & Median & F statistic & p value \\
\hline Debridement with gentamicin-impregnated collagen sponge & $2.00(1.00,3.00)$ & 265.0 & 0.005 \\
Debridement with gentamicin-impregnated PMMA beads & $2.50(2.00,3.75)$ & & \\
\hline
\end{tabular}

PMMA: Polymethylmethacrylate 
Table 2: Comparison of duration of hospitalization period

\begin{tabular}{|c|c|c|c|c|}
\hline Duration hospitalization & DEB+Genta beads+Sysant & Mean & 31.03 & 3.719 \\
\hline & DEB+Collatamp+Systant & $\begin{array}{l}95 \% \text { confidence interval for mean } \\
\text { Lower bond } \\
\text { Upper bond } \\
5 \% \text { trimmed mean } \\
\text { Median } \\
\text { Variance } \\
\text { SD } \\
\text { Minimum } \\
\text { Maximum } \\
\text { Range } \\
\text { Interquartile range } \\
\text { Skewness } \\
\text { Kurtosis } \\
\text { Mean } \\
95 \% \text { confidence interval for mean } \\
\text { Lower bond } \\
\text { Upper bond } \\
5 \% \text { trimmed mean } \\
\text { Median } \\
\text { Variance } \\
\text { SD } \\
\text { Minimum } \\
\text { Maximum } \\
\text { Range } \\
\text { Interquartile range } \\
\text { Skewness } \\
\text { Kurtosis }\end{array}$ & $\begin{array}{l}23.45 \\
38.62 \\
29.56 \\
22.50 \\
442.483 \\
21.035 \\
6 \\
92 \\
86 \\
36 \\
0.931 \\
0.549 \\
23.82 \\
15.39 \\
32.25 \\
21.48 \\
13.50 \\
472.893 \\
21.746 \\
6 \\
87 \\
81 \\
24 \\
1.620 \\
2.139\end{array}$ & $\begin{array}{l}0.414 \\
0.809 \\
4.110\end{array}$ \\
\hline
\end{tabular}

Table 3: CRP trend between the time of diagnosis and at 6 weeks after debridement

\begin{tabular}{|c|c|c|c|c|}
\hline Type of treatment & First CRP & CRP at $6^{\text {th }}$ week & F statistic & p value \\
\hline Debridement with gentamicin-impregnated PMMA beads & $9.411(4.34,12.29)$ & $3.15(1.07,9.67)$ & 0.357 & 0.385 \\
\hline Debridement with gentamicin-impregnated collagen sponge & $8.33(4.39,10.19)$ & $3.25(1.09,5.09)$ & & \\
\hline
\end{tabular}

CRP: C-reactive protein

Table 4: TWC trend between the time of diagnosis and at 6 weeks after debridement

\begin{tabular}{llll}
\hline Type of treatment & First CRP & CRP at 6 $^{\text {th }}$ week $^{\text {F statistic }}$ & p value \\
\hline $\begin{array}{l}\text { Debridement with gentamicin-impregnated PMMA beads } \\
\begin{array}{l}\text { Debridement with gentamicin-impregnated collagen } \\
\text { sponge }\end{array}\end{array}$ & $14.90(11.65,18.55)$ & $9.70(7.38,12.88)$ \\
\hline
\end{tabular}

TWC: Total white cell, CRP: C-reactive protein

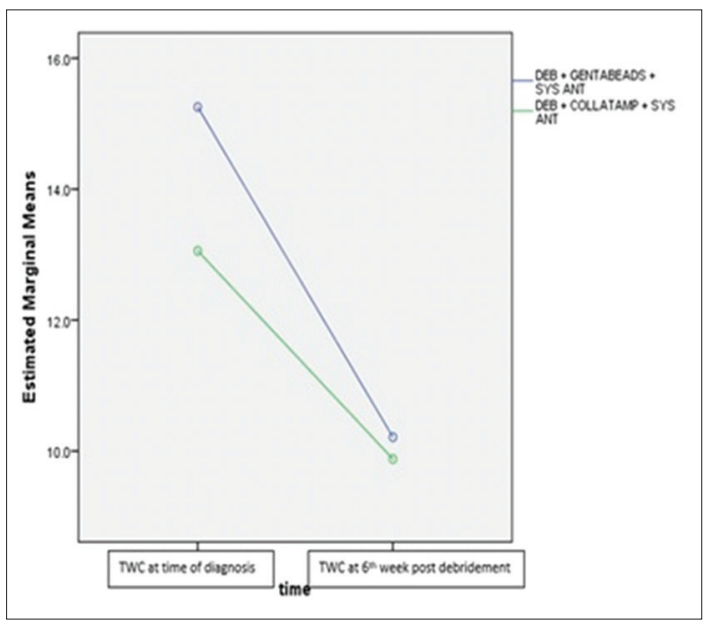

Fig. 3: Total white cell trend between the time of diagnosis and at 6 weeks after debridement

Apart from comparison at the objective period of 6 weeks after debridement, the period for the blood parameters to normalize and stabilize was also compared (Table 5). For CRP, the level is considered normal once it reaches $<0.5 \mathrm{mg} / \mathrm{L}$, whereas the normal level of TWC is taken between the ranges of $4.5-11.0 \times 10^{9} / \mathrm{L}$. "Stabilize" is defined when the blood parameters level is within the normal level for three consecutive blood taking (Table 6).

The collagen sponge group took shorter duration of between 22 and 107 days (mean 50 days) for the CRP to normalize, with the PMMA beads took slightly longer at between 28 and 120 days (mean 62 days). This was, however, not statistically significant ( $\mathrm{p}>0.005)$.

Similarly, comparison of duration for TWC to normalize and stabilize also resulted in a non-significant finding. Although if account for both groups the duration for TWC to normalize is the same (within 7-35 days), patients who underwent debridement with gentamicinimpregnated collagen sponge have shorter duration (mean 13 days) compared to the PMMA beads group (mean 16 days) ( $p>0.05$ ).

Finally, the duration of antibiotics needed to be completed until the resolution of infection also shown no significant difference between the two groups ( $p>0.05$ ), although generally patients who underwent debridement with antibiotic-impregnated collagen sponge completed shorter duration for the antibiotic course (mean 59 days vs. 66 days for PMMA beads group). 
Table 5: Comparison of duration for CRP to normalize and stabilize

\begin{tabular}{|c|c|c|c|c|}
\hline CRP normalized and stabilized & DEB+Genta beads+Sysant & Mean & 62.09 & 4.916 \\
\hline & $x^{2}$ & $\begin{array}{l}\text { 95\% confidence interval for mean } \\
\text { Lower bond } \\
\text { Upper bond } \\
5 \% \text { trimmed mean } \\
\text { Median } \\
\text { Variance } \\
\text { SD } \\
\text { Minimum } \\
\text { Maximum } \\
\text { Range } \\
\text { Interquartile range } \\
\text { Skewness } \\
\text { Kurtosis } \\
\text { Mean } \\
\text { 95\% confidence interval for mean } \\
\text { Lower bond } \\
\text { Upper bond } \\
5 \% \text { trimmed mean } \\
\text { Median } \\
\text { Variance } \\
\text { SD } \\
\text { Minimum } \\
\text { Maximum } \\
\text { Range } \\
\text { Interquartile range } \\
\text { Skewness } \\
\text { Kurtosis }\end{array}$ & $\begin{array}{l}52.07 \\
72.12 \\
60.79 \\
54.50 \\
773.314 \\
27.809 \\
28 \\
120 \\
92 \\
36 \\
0.842 \\
-0.451 \\
50.11 \\
\\
40.55 \\
59.66 \\
48.52 \\
42.00 \\
607.433 \\
24.646 \\
22 \\
107 \\
85 \\
26 \\
1.210 \\
0.643\end{array}$ & $\begin{array}{l}0.414 \\
0.809 \\
4.658\end{array}$ \\
\hline
\end{tabular}

Table 6: Comparison of duration for TWC to normalize and stabilize

\begin{tabular}{|c|c|c|c|c|}
\hline TWC normalized and stabilized & DEB + Genta beads + Sysant & Mean & 16.28 & 1.352 \\
\hline & DEB + Collatamp + Systant & $\begin{array}{l}\text { 95\% confidence interval for mean } \\
\text { Lower bond } \\
\text { Upper bond } \\
5 \% \text { trimmed mean } \\
\text { Median } \\
\text { Variance } \\
\text { SD } \\
\text { Minimum } \\
\text { Maximum } \\
\text { Range } \\
\text { Interquartile range } \\
\text { Skewness } \\
\text { Kurtosis } \\
\text { Mean } \\
\text { 95\% confidence interval for mean } \\
\text { Lower bond } \\
\text { Upper bond } \\
5 \% \text { trimmed mean } \\
\text { Median } \\
\text { Variance } \\
\text { SD } \\
\text { Minimum } \\
\text { Maximum } \\
\text { Range } \\
\text { Interquartile range } \\
\text { Skewness } \\
\text { Kurtosis }\end{array}$ & $\begin{array}{l}13.52 \\
19.04 \\
15.80 \\
14.00 \\
58.467 \\
7.646 \\
7 \\
35 \\
28 \\
10 \\
1.121 \\
0.406 \\
13.29 \\
10.83 \\
15.74 \\
12.65 \\
11.50 \\
40.138 \\
6.335 \\
7 \\
35 \\
28 \\
8 \\
1.617 \\
3.732\end{array}$ & $\begin{array}{r}0.414 \\
0.809 \\
1.197\end{array}$ \\
\hline
\end{tabular}

\section{DISCUSSION}

Resolution of infection and prevention of recurrent chronic osteomyelitis remains as the major goals of the treatment of osteomyelitis. The two mainstay of treatment is an adequate debridement and commencement of systemic antibiotics. However, the role of usage of local antibiotics as adjunct should not be dismissed. Started with prophylactic measure in prosthetic joint surgery, the introduction of antibiotic-impregnated beads later took center stage and mainly used as adjunct to surgical debridement to reduce the incidences of post-operative infection $[6,7]$. The introduction of gentamicin-impregnated beads reported a cure rate of $91.4 \%$ in over 100 patients [8].

One of the main advantages of antibiotic-impregnated medium is the delivery of high concentration of antibiotics to the local tissue affected by the infection. This is in contrast with the poor delivery of systemic antibiotics due to the disruption of blood supply, especially in chronic osteomyelitis. The presence of biofilm is also a major factor as it acts 
as mechanical barrier for the delivery of systemic antibiotics. Hence, high level of antibiotic concentrations is required to penetrate biofilms formed by the offending organism [9]. Mostly, the systemic antibiotics given in standard, regular doses are insufficient to breach the glycocalyx produced by the offending bacteria [10]. Thus, delivery of high local concentration of antibiotics can target the remaining organisms in biofilms more effectively [11]. Local antibiotics may act as a gap filler and help bathing the hematoma or seroma with antibiotics. The dead space that may be left after debridement was shown to be effectively occupied by antibiotic beads [12]. The framework consisting of osteoinductive and osteoconductive properties can be provided by the local antibiotics, aiding in bone healing following osteomyelitis [13].

One of the methods for local antibiotic delivery which is widely used is the usage of antibiotic-impregnated PMMA beads. It was proposed that cement beads are the gold standard for local antibiotic delivery for orthopedic infection treatment and prophylaxis [11]. Antibiotic consignment from PMMA beads also produces significantly higher concentration levels in targeted tissue, as compared than systemic antibiotic therapy alone [14]. On the other hand, the relatively new antibiotic-impregnated collagen sponge has also been shown to be an effective adjunct for the treatment of osteomyelitis $[15,16]$. Due to its flexible form, it may fit into small spaces at the wound following the debridement, hence, provided a better alternative than solid beads with chains. However, more studies are needed in regard to the efficacy of gentamicin-impregnated collagen sponge as the treatment for osteomyelitis as most studies were focusing on its efficacy as prophylaxis for surgical site infection, especially in cardiothoracic and colorectal surgeries $[17,18]$.

As in many discussions regarding choice of antibiotic for local delivery, gentamicin had been proven as an excellent choice. Among the most common pathogens isolated in osteomyelitis are Staphylococcus sp. and Pseudomonas sp., with Staphylococcus epidermidis are notably notorious in the presence of implant [19]. As concentration-dependent aminoglycosides are effective against both Gram-positive and Gramnegative bacteria $[20,21]$, gentamicin provides a useful bactericidal effect. A potent additive to any local antibiotic carrier, gentamicin sulfate itself has shown to have low rate of primarily resistant pathogens, in addition, to have good thermostability [22]. This is definitely important, especially when mixed with the exothermic-producing PMMA beads.

The issue of safety of high concentration of local delivery of gentamicin, however, is still inconclusive and may require more studies. Although the concentration used in this study is generally low (135 mg per one collagen sponge and $135 \mathrm{mg}$ per one PMMA beads chain), there are studies that highlight concerns regarding the adverse effects of local delivery of highly concentrated antibiotics. A recent animal study found that the usage of antibiotic-impregnated collagen sponge led to the development of renal failure in dogs [23]. Whereas the usage of topical gentamicin-impregnated collagen sponge in diabetic foot ulcers showed that $28.6 \%$ of sample developed adverse effects, namely elevated serum creatinine level, infected skin ulcers, and tinea pedis infection [24].

Due to the differences in form and requirements, in vitro comparative studies between antibiotic-impregnated PMMA beads and collagen sponge have been conducted and found that the bacterial colony was significantly reduced in rats treated with gentamicin with collagen sponge, while both significantly better than only parenteral cefazolin. Gentamicin-impregnated collagen sponge was also shown to produce higher minimal inhibitory concentration (MIC) as compared to PMMA beads (almost 600 times MIC for the collagen sponge vs. 300 times MIC for cement beads). Therefore, the relation of release of large amounts of gentamicin, the flexible gentamicin-contained collagen sponge proved to be superior to the rigid PMMA beads [25].

Studies comparing the clinical outcomes in patients diagnosed with osteomyelitis and managed with antibiotic PMMA beads and collagen sponge, however, are still small in number. The previous study found that $80 \%$ of the collagen sponge group and in $90 \%$ of the PMMA beads group the osteomyelitis was completely allayed with disappearance of all infectious parameters. There was significantly higher reoperations number in the PMMA group. It was also found out that gentamicin is released more rapidly in collagen sponge group as compared to the PMMA beads group [26].

This retrospective study attempted to compare the measurable outcomes among these patients. The two statistically significant findings were the collagen sponge group that has underwent lower numbers of debridement and shown to have significant reduction of TWC count as compared to the cement beads group. The lower reoperative rate mainly owes to the fact that majority of patients with gentamicin-impregnated PMMA beads required a second, though simpler, surgery to remove the beads, especially for those whose wounds were closed during the first debridement. In comparison, the gentamicin-impregnated collagen sponge does not require a second surgery to remove it, as it is biodegradable. The lower reoperative rate shown in this study also tallies with previous studies.

Therefore, among the drawbacks for the usage of PMMA beads are the need for another surgery to remove the beads after sometime. After the concentration of gentamicin released from the local carrier diminishes, the beads and the chain will only serve to become a foreign body. Hence, it becomes a risk to provide a reservoir for organisms to survive and recolonize. PMMA beads may also induce undesirable local tissue reaction and require large space to be inserted effectively [27]. Although there are still practices that leave the end of the chain protruding out from part of the wound that is left open following insertion of the beads, this is not routinely done, especially in periarticular and juxtaarticular osteomyelitis. The need for removal of the PMMA beads is a major factor found in our study on why the reoperative rate is higher as compared to the patients who underwent debridement using gentamicin-impregnated collagen sponge.

The significant reduction of the TWC count in patients who underwent debridement with antibiotic-impregnated collagen sponge is a new finding. At first, we have hypothesized that there will be significant reduction of all blood parameters, especially the CRP in the collagen sponge group due to lower number of debridement. This is despite previous studies investigating the biodegradation of the collagen sponge following its subcutaneous and intramuscular implantation have recognized a pattern of significant inflammatory response lingering for at least $120 \mathrm{~h}$ after the application [28,29]. As repeated surgical debridement will induce more tissue reaction and inflammation, the PMMA beads were hypothesized to have much slower reduction in inflammatory markers level. However, the study showed that there was no difference in terms of reduction of the CRP, thus dispelling the hypothesis. Although TWC is also considered as a reliable clinical parameter for inflammation and infection, CRP is a more sensitive and accurate measure $[30,31]$.

As postulated, there were no significant differences in hospitalization period and duration of systemic antibiotics to be completed in both groups of patients. This is consistent with previous studies and showed that despite the usage of local delivery of antibiotics, systemic antibiotics remain the mainstay of treatment and must not be compromised. As host factor remains as the most important factor that has critical importance in inducing remission of chronic osteomyelitis, the susceptibility to infection need to be countered with a good, effective systemic antibiotics commencement [32]. The standard recommendation of 6 weeks of antibiotics for the treatment of chronic osteomyelitis is still widely accepted. Studies have shown that intravenous and oral therapies have achieved similar curative rate, and there are no data of antibiotic therapy more than 6 weeks will improve the clinical outcomes [33].

This study has been rightly shown to have few limitations; among the notable one is the study design itself. Like many retrospective 
studies, the inability to control variable biases, especially in regard to patients' factors, may influence the outcome of this study. As this may be considered as a pilot study, the limited numbers of published studies comparing the two groups of gentamicin-impregnated collagen sponge and PMMA beads provide limited exposure and references to ensure the suitability of the clinical outcomes tested. A randomized, prospective study with greater number of sample size using similar points for comparison would be a good follow-up to ascertain the accuracy of the findings in this study.

\section{CONCLUSIONS}

This study reinforces previous studies' findings that the usage of gentamicin-impregnated collagen sponge will result in lower reoperative rate as compared to conventional gentamicin-impregnated PMMA beads. The significant reduction of TWC count, however, may need further comprehensive studies, especially the ones that would focus on assessing the reduction of inflammatory parameters as an indicator for the response of treatment in patients with osteomyelitis who undergoes debridement with application of gentamicinimpregnated collagen sponge. The study also reiterates the efficacy of usage of gentamicin-impregnated sponge as adjunct of treatment in osteomyelitis, in relative comparison to the usage of PMMA beads which is considered as the gold standard for local antibiotic delivery in osteomyelitis treatment.

\section{AUTHORS' CONTRIBUTION}

Ahmad Arief Atan and Ahmad Fazly Abd Rashid carried out the experiment. Ahmad Arief Atan and Abdul Muhaimin Ali wrote the manuscript with support from Rasyidah Rehir. Ahmad Arief Atan and Mohd Yazid Bajuri conceived the original idea. Mohd Yazid Bajuri supervised the project.

\section{CONFLICTS OF INTEREST}

All authors have none to declare.

\section{REFERENCES}

1. Malizos KN, Kirketerp-Moller K. Incidence and socioeconomic impact of bone and joint infections (BJIs): The European perspective. In: Periprosthetic Joint Infections, Part 1. Switzerland: Springer; 2016. p. 3-18.

2. Poultsides LA, Liaropoulos LL, Malizos KN. The Socioeconomic impact of musculoskeletal infections. J Bone Joint Surg Am 2010;2:e13.

3. Lowenberg DW. A technotheoretical approach to the management of osteomyelitis. Tech Orthop 2015;30:209-14.

4. Beaudoin T, Waters V. Infections with biofilm formation: Selection of antimicrobials and role of prolonged antibiotic therapy. Paed Infect Dis J 2016;35:695-7.

5. Cierny G. Surgical treatment of osteomyelitis. Plast Reconstr Surg 2011;127:190S-204.

6. Buchholz HW, Engelbrecht H. Depot effects of various antibiotics mixed with Palacos resins (translated). Chirurg 1970;41:511-5.

7. Kala P, Rani RJ, Sangeetha R. Prescription pattern of prophylactic antimicrobial agents used in preoperative patients at a tertiary care teaching hospital. Int J Pharm Pharm Sci 2018;10:128-31.

8. Klemm K. Gentamicin-PMMA-beads in treating bone and soft tissue infections (author's translation). Zentralbl Chir 1979;104:934-42.

9. Nelson CL. The current status of material used for depot delivery of drugs. Clin Orthop Relat Res 2004;427:72-7.

10. El-Husseiny M, Patel S, MacFarlane RJ, Haddad FS. Biodegradable antibiotic delivery systems. J Bone Joint Surg Br 2011;93:71.

11. Hanssen AD. Local antibiotic delivery vehicles in the treatment of musculoskeletal infection. Clin Orthop Related Res 2005;437:91-6.

12. Patzakis MJ, Mazur K, Wilkins J, Sherman R, Holtom P. Septopal beads and autogenous bone grafting for bone defects in patients with chronic osteomyelitis. Clin Orthop Relat Res 1993;295:112-8.

13. Gitelis S, Brebach GT. The treatment of chronic osteomyelitis with a biodegradable antibiotic impregnated implant. J Orthop Surg 2002; 10:53-60.

14. Hoff SF, Fitzgerald RH Jr., Kelly PJ. The depot administration of penicillin $\mathrm{g}$ and gentamicin in acrylic bone cement. J Bone Joint Surg Am 1981;63:798-804.

15. Ipsen T, Jorgensen PS, Damholt V, Torholm C. Gentamicin-collagen sponge for local applications: 10 cases of chronic osteomyelitis followed for 1 year. Acta Orthop Scand 1991;62:592-4.

16. Kanellakopoulou K, Galanakis N, Giamarellos-Bourboulis EJ, Rifiotis C, Papakostas K, Andreopoulos A, et al. Treatment of experimental osteomyelitis caused by methicillin-resistant Staphylococcus aureus with a biodegradable system of lactic acid polymer releasing pefloxacin. J Antimicrob Chemother 2000;46:311-4.

17. Chang WK, Srinivasa S, MacCormick AD, Hill AG. Gentamicincollagen implants to reduce surgical site infection: Systematic Review and meta-analysis of randomized trials. Ann Surg 2013;258:59-65.

18. Bennett-Guerrero E, Pappas TN, Koltun WA, Fleshman JW, Lin M, Garg J, et al. Gentamicin-collagen sponge for infection prophylaxis in colorectal surgery. N Engl J Med 2010;363:1038-49.

19. Ciampolini J, Harding KG. Pathophysiology of chronic bacterial osteomyelitis: Why do antibiotics fail so often? Postgrad Med J 2000;76:479-83.

20. Tam VH, Kabbara S, Vo G, Schilling AN, Coyle EA. Comparative pharmacodynamics of gentamicin against Staphylococcus aureus and Pseudomonas aeruginosa. Antimicrob Agents Chemother 2006;50:2626-31.

21. Kumar SL, Ashutosh SR, Gokulshankar S, Ranjith MS, Mohanty BK, Lim MY. Is bacteriology a contributing factor in unsalvageable nature of diabetic foot infections?-a study in a district hospital in Malaysia. Int J Pharm Pharm Sci 2015;8:262-5.

22. Klemm K. The use of antibiotic-containing bead chains in the treatment of chronic bone infections. Clin Microbiol Infect 2001;7:28-31.

23. Hayes G, Gibson T, Moens NM, Nykamp S, Wood D, Foster R, et al. Intra-articular implantation of gentamicin impregnated collagen sponge causes joint inflammation and impaired renal function in dogs. Vet Comp Orthop Traumatol 2016;29:159-63.

24. Lipsky BA, Kuss M, Edmonds M, Reyzelman A, Sigal F. Topical application of a gentamicin-collagen sponge combined with systemic antibiotic therapy for the treatment of diabetic foot infections of moderate severity: A randomized, controlled, multicenter clinical trial. J Am Podiatr Med Assoc 2012;102:223-32.

25. Mendel V, Simanowski HJ, Scholz HC, Heymann H. Therapy with gentamicin-PMMA beads, gentamicin-collagen sponge, and cefazolin for experimental osteomyelitis due to Staphylococcus aureus in rats. Arch Orthop Trauma Surg 2005;125:363-8.

26. Letsch R, Rosenthal E, Joka T. Local antibiotic application in the treatment of osteomyelitis - A comparative study of two different carriers (translated). Aktuelle Traumatol 1993;23:324-9.

27. Tsourvakas S. Local antibiotic therapy in the treatment of bone and soft tissue infections. In: Selected Topics in Plastic Reconstructive Surgery. Croatia: In Tech; 2012. p. 18-34.

28. Mehta S, Humprey JS, Schenkman DI, Seaber AV, Vail TP. Gentamicin distribution from a collagen carrier. J Orthop Res 1996;14:749-54.

29. Kilian O, Hossain H, Flesch I, Sommer U, Nolting H, Chakraborty T, et al. Elution kinetics, antimicrobial efficacy, and degradation and microvasculature of a new gentamicin-loaded collagen fleece. J Biomed Mater Res B Appl Biomater 2009;90:210-22.

30. Clyne B, Olshaker JS. The C-reactive protein. J Emerg Med 1999; 17:1019-25.

31. Pepys MB, Hirschfield GM. C-reactive protein: A critical update. J Clin Invest 2003;111:1805-12.

32. Blaha JD, Calhoun JH, Nelson CL, Henry SL, Seligson D, Esterhai JL, et al. Comparison of the clinical efficacy and tolerance of gentamicin PMMA beads on surgical wire versus combined and systemic therapy for osteomyelitis. Clin Orthop Relat Res 1993;295:8-12.

33. Spellberg B, Lipsky BA. Systemic antibiotic therapy for chronic osteomyelitis in adults. Clin Infect Dis 2012;54:393-407. 